\title{
EL PALO ESCRITO, ARBOL DE MADERA PRECIOSA - UNA NUEVA ESPECIE MEXICANA DE DALBERGIA (LEGUMINOSAE, PAPILIONOIDEAE)'
}

\author{
JERZYRZEDOWSKI \\ Instituto de Ecología, Centro Regional del Bajío \\ Apartado postal 386; 61600 Pátzcuaro, Mich. México \\ LYDIA I. GURIDI-Gomez \\ Escuela de Ingeniería en Tecnología de la Madera \\ Universidad Michoacana de San Nicolás de Hidalgo \\ 58060 Morelia, Mich. México
}

\begin{abstract}
RESUMEN
Se describe Dalbergia palo-escrito sp. n., planta endémica del bosque mesófilo de montaña de la Sierra Madre Oriental del estado de Hidalgo, así como de zonas adyacentes de San Luis Potosí y Querétaro. La especie parece estar cercana a D. tucurensis Donn. Smith, conocida de Belice y Chiapas a Nicaragua. Se proporciona la descripción anatómica de la madera.
\end{abstract}

\section{ABSTRACT}

Dalbergia palo-escrito sp. $n$. is described from the montane mesophyllous forest of the Sierra Madre Oriental in the state of Hidalgo and in adjacent parts of San Luis Potosi and Querétaro. It seems to be related to $D$. tucurensis Donn. Smith, known from Belize and Chiapas to Nicaragua. An anatomical description of the wood is provided.

En la región escarpada de la Sierra Madre Oriental, correspondiente al rincón limítrofe de los estados de Querétaro, San Luis Potosí e Hidalgo es de mucho renombre y uso la madera de "palo escrito", árbol nativo, que aún se encuentra con bastante abundancia, a pesar de la intensa modificación que ha sufrido la cubierta vegetal de la comarca.

El área en cuestión se localiza en la inmediata vecindad de la región de la Huasteca y para algunos forma parte de la misma; no es de extrañarse, por consiguiente, que el palo escrito tenga también su nombre propio en el idioma huasteco al igual que en el náhuatl local.

Al parecer, la primera noticia publicada sobre esta planta se encuentra en los trabajos de Rzedowski (1965, pp. 66, 88, 198, 200; 1967, pp. 22, 49, 50), mencionada como Dalbergia sp. Por otra parte, Guridi Gómez $(1980, \mathrm{pp} .114,115)$ ilustra una guitarra fabricada en Paracho, Mich. con varias partes elaboradas de palo escrito.

Las particularidades de la madera de este árbol son similares a las de muchos otros representantes de Dalbergia; igual cosa sucede con las características de la inflorescencia, de la flor, del fruto y de las hojas, por lo cual no hay duda acerca de su ubicación genérica.

Dalbergia, a su vez, es un taxon que comprende un centenar o más de especies leñosas, distribuidas en las zonas tropicales de ambos hemisferios y es un hecho desafortunado

'Trabajo realizado con apoyo del Consejo de Ciencia y Tecnología del Estado de Querétaro, del Centro de Investigación y Desarrollo del Estado de Michoacán y del Consejo Nacional de Ciencia y Tecnología. 
que desde los tiempos de Bentham (1860) el grupo no ha sido taxonomicamente revisado. Muchas de las especies descritas se conocen sólo de manera fragmentaria y se ignora la magnitud de su variabilidad morfológica, así como su distribución geográfica y sus afinidades ecológicas precisas.

En tal virtud, resulta un tanto aventurado distinguir una especie más de este género, pero después de consultar los trabajos de Pittier (1922), de Standley (1922, 1937), de Standley y Steyermark (1946) y de Dwyer (1965) así como las descripciones de todas las especies de Dalbergia citadas para México y Centroamérica, se llega a la conclusión de que el palo escrito, aun sin ser muy diferente, no se ajusta a las características de ninguna. En consecuencia se le propone como

\section{Dalbergia palo-escrito sp. n. (Fig. 1)}

Arbor sempervirens, usque ad $35 \mathrm{~m}$ alta, partibus viridibus albido- vel ferrugineopuberulis, aliquis plus minusve glabrescentibus; foliola (5)9-13, (3)4-7 cm longa, (1)2-3.5 cm lata, ovata, supra atroviridia, subtus conspicue pallidiora; inflorescentiae axillares, paniculatae, usque ad $5 \mathrm{~cm}$ longae et latae; flores $3-5.5 \mathrm{~mm}$ longi, albidi vel luteoli, calyx insigniter zygomorphus, corollae longitudinis dimidium aequans, vexillum erectum, obovatum, stamina 9 , ovarium stipitatum, stylo crasso, $\pm 1 \mathrm{~mm}$ longo; fructus monospermus, oblongus, $4-7.5 \mathrm{~cm}$ longus, $1-1.5 \mathrm{~cm}$ latus, brunneus, puberulus. $D$. tucurensi Donn. Smith ut videtur affinis, sed ab ea foliolis ovatis, pubescentia lutea carentibus, et legumine pubescenti discrepans.

Arbol perennifolio hasta de $35 \mathrm{~m}$ de alto, tronco hasta de $80 \mathrm{~cm}$ de diámetro, ramillas jóvenes antrorsa y aplicadamente pubérulas con pelos rojizos o blanquecinos de 0.1 a $0.2 \mathrm{~mm}$ de largo, las de años anteriores grisáceas, glabrescentes.

Hojas (5)9-13-folioladas; estípulas muy pronto caedizas; peciolo y raquis densamente pubérulos en la juventud, glabros o casi glabros con la edad, peciólulos de 2 a $3 \mathrm{~mm}$ de largo, densamente hispídulos, al menos en la juventud, el peciólulo del foliolo terminal inserto sobre una prolongación del raquis de $\pm 1 \mathrm{~cm}$ de largo, más delgada y de estruc tura diferente de lo que propiamente parece ser el peciólulo; foliolos angosta a anchamente ovados, de (3)4 a $7 \mathrm{~cm}$ de largo y (1)2 a $3.5 \mathrm{~cm}$ de ancho, ápice redondeado, a menudo levemente retuso, base por lo general redondeada a subtruncada (en el foliolo terminal con frecuencia cuneada), a menudo asimétrica, de textura membranácea, nervadura central prominente en el envés, las laterales principales 10 a 12 pares, inás conspicuas en el haz, desprendiéndose en ángulo de 50 a $60^{\circ}$, en el envés cabe observar un fino retículo de nervaduras de último orden, haz de color verde olivo oscuro, envés verde claro, algo grisáceo, ambas caras con puberulencia aplicada poco conspicua.

Inflorescencia en forma de panículas axilares, moderadamente densas a laxas, hasta de $5 \mathrm{~cm}$ de largo y de diámetro, sus ejes densamente ferrugineo-pubérulos con pelos aplicados de 0.1 a $0.2 \mathrm{~mm}$ de largo, brácteas y bracteolas de $1 \mathrm{~mm}$ o menos de largo, densamente pubérulas, lanceoladas a lineares, pedicelos de 1 a $2 \mathrm{~mm}$ de largo; flores de 3 a $5.5 \mathrm{~mm}$ de largo, de oior poco agradable; cáliz campanulado, notablemente zigomórfico, de la mitad de la longitud de la flor o a veces un poco más largo, densamente ferrugineo-pubérulo, el lóbulo impar hasta $1 \mathrm{~mm}$ más largo que los demás, los lóbulos superiores unidos entre sí hasta 4/5 de su largo; corola blanquecina a amarillenta, glabra, estandarte erecto, con la lámina obovada, 
Rzedowski y Guridi-Gómez: El Palo Escrito, Nueva Especie Mexicana de Dalbergia

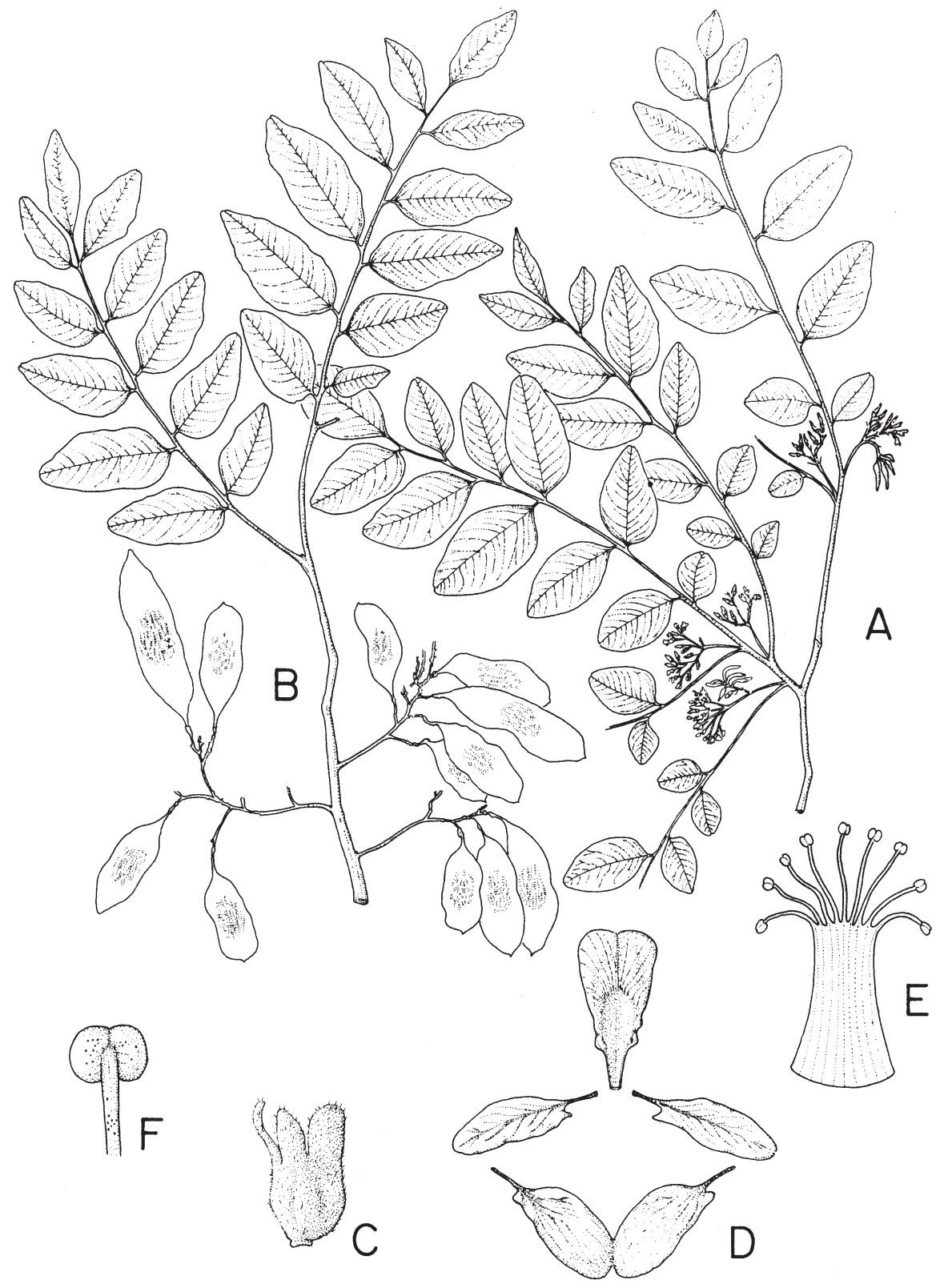

Fig. 1. Dalbergia palo-escrito Rzedowski \& Guridi-Gómez. A. Rama con flores; B. Rama con frutos; C. Cáliz; D. Pétalos, E. Androceo; F. Antera. 
emarginada en el ápice, subbiauriculada en la base, la uña de $\pm 1 / 4$ del largo total del pétalo, alas libres de la quilla, aproximadamente del largo del estandarte, su lámina oblonga, auriculada o subauriculada en la base de un solo lado o de ambos lados, los pétalos de la quilla similares, pero un poco más anchos y auriculados de un solo lado; estambres 9 , monadelfos, el tubo glabro: ovario estipitado, densamente pubérulo, estilo grueso, de $\pm 1 \mathrm{~mm}$ de largo, estigma terminal, oblicuo.

Fruto oblongo, de 4 a $7.5 \mathrm{~cm}$ de largo, de 1 a $1.5 \mathrm{~cm}$ de ancho, redondeado u obtuso y a veces mucronado en el ápice, cuneado en la base, pasando en forma más o menos abrupta al delgado estípite, monospermo, de color café, con la venación prominentemente reticulada en el área correspondiente a la semilla y de manera menos conspicua en el resto del fruto, puberulencia similar a la de las hojas.

TIPO: México, Querétaro, La Parada, municipio de Jalpan, alt. $1100 \mathrm{~m}$, terreno plano con vegetación de bosque mesófilo de montaña con Liquidambar, orilla de camino, 23.VI.1988, S. Zamudio 5616 (ENCB, holotipo).

Material adicional examinado: SAN LUIS POTOSI, Ahuacatlán. municipio de Xilitla, alt. 1250 m, 11.IV.1960, "escrito", Rzedowski 12398 (fl) (IEB). QUERETARO, 3 km al SE de Agua Zarca, municipio de Landa, sobre el camino a Pisaflores, alt. 1350m, 16.III.1987, "palo escrito", Rzedowski 42837 (fl) (IEB); al sureste del Rincón de Peña Blanca, municipio de Landa, alt. 1300 m, 12.IX.1988, "escrito", H. Rubio 138 (fr) (IEB). HIDALGO, km 327 on highway between Santa Ana and Chapulhuacán, 12.VII.1948, H.E. Moore \& C.E. Wood, Jr. 3980 (fr) (MEXU); $3 \mathrm{~km}$ al NNE de Chapulhuacán, alt. 1400 m, 9.IV.1960, "palo escrito", Rzedowski 12350 (fl) (IEB); alrededores de Chapulhuacán, 25.VI.1960, "palo escrito", J. Márquez s.n. (fr) (IEB); Santa María, municipio de Chapulhuacán, alt. $\pm 1000 \mathrm{~m}, 14$.VI.1975, "palo escrito", M. Seidels.n. (fr) (MEXU); 4 km al NE de Cuesta Colorada, municipio de Jacala, alt. $1900 \mathrm{~m}, 12.1 \mathrm{l} .1982, R$. Hernández M. \& P. Tenorio L. 6959 (fl) (MEXU); carretera Ixtlahuaco, tramo a Calmali, municipio de Calmali, alt. 1500 m, 8.VIII.1976, "tlanchinol", J.I. Calzada 2504 (fr) (MEXU); Molango, 12.VIII.1961, "tlacuilo", L. Vela \& X. Madrigal229(fr) (ENCB, MEXU); 15.6 km al N de Tianguistengo, carretera a Molango, 22.VI.1983, R. Torres \& H. Hernández 3077 (fr) (MEXU); $4.8 \mathrm{~km}$ al N de Tianguistengo, hacia Molango, 22.VI.1983, R. Torres \& H. Hernández 3074 (fr) (MEXU); $4 \mathrm{~km}$ al N de Tianguistengo, hacia Xochicoatlán, alt. $1600 \mathrm{~m}, 26.111 .1981, R$. Hernández M. \& D. Rodriguez 5659 (fl) (ENCB, MEXU); $3 \mathrm{~km}$ al NE de Tianguistengo (hacia la barranca de Chinameca), alt. $1700 \mathrm{~m}, 5.111 .1982$, P. Tenorio L. \& R. Hernández 122 (fl) (MEXU).

Descripción anatómica de la madera

La corteza externa es rugosa, de color castaño amarillento claro, está cubierta con musgos y líquenes pequeños y abundantes, que le confieren color verde a la superficie; tiene aproximadamente $0.2 \mathrm{~cm}$ de espesor. La corteza interna es de color crema y tiene $0.4 \mathrm{~cm}$ de grosor.

La madera presenta marcada diferencia de color entre la albura, que es de color crema, y el duramen castaño violáceo con vetas más oscuras, casi negras, que en las caras transversales semejan anillos de crecimiento de contorrio muy irregulary son los responsables de su hermoso y vistoso veteado (Fig. 2). El olor y sabor no se perciben, tiene veteado 
Rzedowski y Guridi-Gómez: El Palo Escrito, Nueva Especie Mexicana de Dalbergia

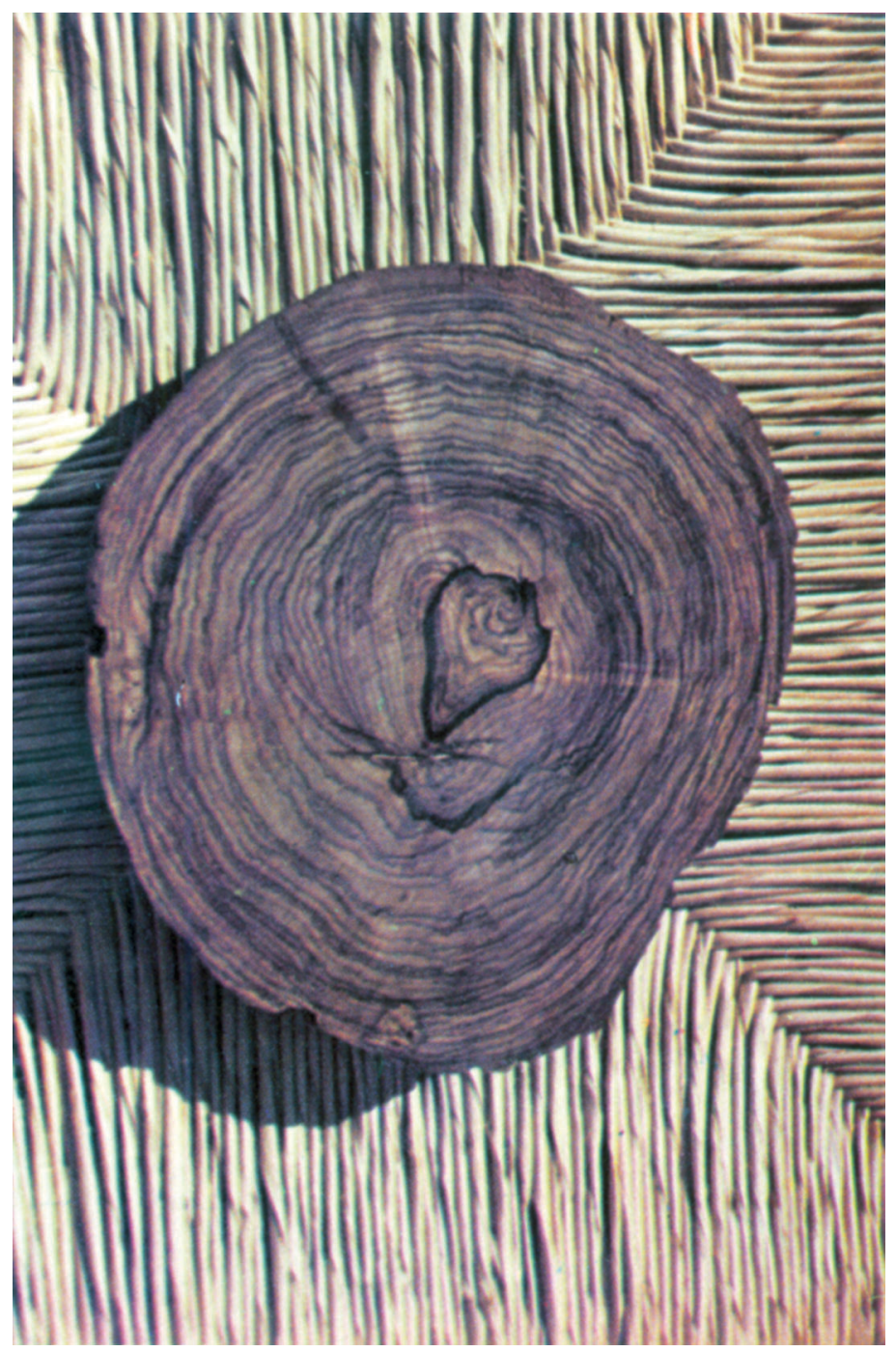

Fig. 2. Dalbergia palo-escrito Rzedowski \& Guridi-Gómez. Corte transversal del tronco de un individuo joven. 
pronunciado, textura mediana, hilo recto y brillo medio. Es muy dura y pesada, no difícil de trabajar y su acabado es fino y pulido. Los anillos de crecimiento no se presentan, pero existe cierta zonación debido a la presencia de parénquima terminal. La porosidad es difusa, con ligera tendencia a circular. Los poros visibles a simple vista, bastante conspicuos en las caras tangenciales, son de pequeños a grandes, predominantemente solitarios, en grupos radiales de 2, ocasionalmente de 3 y escasos en grupos irregulares. El parénquima leñoso visible con lupa, es terminal y paratraqueal vasicéntrico, de pocas células de ancho. Los rayos sólo visibles con lupa, son muy notables y numerosos y resaltan por su color más claro en las caras transversales.

Nombres comunes

El nombre "palo escrito" o "escrito" prevalece en la actualidad para la especie en el área correspondiente a los estados de Querétaro y San Luis Potosí así como a zonas inmediatamente adyacentes de Hidalgo. Es probable que el término se originó en función de la coloración veteada de la madera. En la misma zona están en uso también las denominaciones "tlajuilocuáhuitl" (náhuatl) y "tzipil", "tzipilín" (huasteco).

Los nombres recogidos en la región de Molango son "tlacuilo" y "tlanchinol". El primero es, sin duda, una variante de tlajuilocuáhuitl. Del segundo probablemente deriva el que corresponde al poblado de Tlanchinol, una de las cabeceras municipales de la comarca. Sin embargo, sería recomendable corroborar si efectivamente se aplica al árbol en cuestión.

Usos

La madera, por su hermoso color, contrastante veteado y propiedades de resonancia, es muy apreciada por los artesanos de Michoacán (Paracho), quienes la utilizan en el fondo y en la costilla de guitarras de tipo clásico, las cuales se cotizan a alto precio en el mercado por su gran demanda.

En la región limítrofe de los estados de San Luis Potosí, Querétaro e Hidalgo, municipios de Aquismón, Xilitla, Tamazunchale, Jalpan, Landa, Chapulhuacán y Pisaflores, el palo escrito se emplea principalmente para trabajos de ebanistería, siendo superior su demanda y su precio a los de la madera de cedro rojo (Cedrela odorata).

Es de llamar la atención, por consiguiente, que en la zona de Molango y Tianguistengo, del estado de Hidalgo, donde el árbol también es más o menos común, aparentemente no se le utiliza con fines de construcción de muebles, pues la única indicación de uso en las etiquetas de los ejemplares examinados lo describe como árbol de sombra. Resulta notable asimismo que el nombre "palo escrito" no esté en uso ahí.

Por otra parte es pertinente señalar que muchos otros representantes de Dalbergia también tienen características similares. De acuerdo con Record y Hess (1943), unas 15 especies, de diferentes partes del mundo, son objeto de comercio internacional por las propiedades tecnológicas favorables de su madera, combinadas con su colorido y a menudo también con un agradable aroma. Entre éstas destacan D. nigra Fr. Allem. del Brasil (palisandro), $D$. retusa Hemsl. y especies afines de Centroamérica (cocobolo del comercio), D. sissoo Roxb. de la India (sissoo) y D. melanoxylon G. \& P. de Africa (Senegal ebony), este último usado sobre todo para la elaboración de instrumentos musicales. Excelentes 
características acústicas presenta también D. stevensonii Standl. de Belice (Honduras rosewood), que se emplea para la fabricación de marimbas y xilófonos.

Distribución geográfica y afinidades ecológicas

D. palo-escrito se muestra como una especie endémica de un corto segmento de la Sierra Madre Oriental (entre latitudes de $20^{\circ} 40^{\prime}$ y $21^{\circ} 30^{\prime} \mathrm{N}$ ), incluyendo reducidas porciones de los estados de Querétaro y San Luis Potosí y una fracción más amplia de Hidalgo.

El árbol está claramente vinculado con el bosque mesófilo de montaña de la región y se le encuentra de preferencia entre 900 y 1700 m s.n.m.; sólo una localidad indica el bosque de Juniperus y Pinus, a $1900 \mathrm{~m}$ de altitud. Al parecer el substrato geológico de todas las poblaciones lo constituyen rocas sedimentarias marinas, mayormente calizas.

Como ya se indicó con anterioridad, el área de distribución de esta especie corresponde a una comarca intensamente perturbada por la agricultura más o menos transhumante y por la ganadería intensiva. La vegetación en general se halla profundamente modificada y sólo en algunas localidades de difícil acceso se localizan vestigios del bosque primitivo.

En el área próxima a Chapulhuacán y Xilitla, el palo escrito formaba parte del bosque mesófilo de montaña original como elemento relativamente poco importante desde el punto de vista cuantitativo. Es ahí donde se le puede encontrar en forma de árbol hasta de $35 \mathrm{~m}$ de alto.

Al igual, sin embargo, que Liquidambar styraciflua, Dalbergia palo-escrito resulta sin duda favorecida por ciertos tipos de disturbio y con frecuencia abunda en los bosquetes secundarios, salpicados a manera de manchones por toda la región. También se nota que el hombre a menudo favorece su presencia, pues es frecuente encontrarla en cercas e inclusive en huertos familiares. Se trata casi siempre en estos casos de árboles relativamente pequeños - arbustos. En el área cercana a Molango la situación también parece ser similar.

Fenología y reproducción

Aun cuando se trata de una especie siempre verde, al tiempo de la floración o poco antes acontece una substitución del follaje.

Las flores se observan entre febrero y abril; ejemplares con frutos se han colectado de junio a septiembre. En ninguno de los especímenes examinados, sin embargo, se detectaron semillas bien desarrolladas. Parece ser tan drástico este fenómeno que los intentos de reproducir los árboles a través de viveros se han visto frustrados por la escasez de semillas (Mauro Márquez, com. pers.). La larva de un insecto (probablemente de la familia Bruchidae) es la responsable del consumo masivo de estos propágulos.

El problema amerita, desde luego, de un estudio más profundo, pues no es posible que sin formar semillas viables, la especie se reproduzca en forma natural tan bien como para constituirse en uno de los elementos arbóreos más abundantes en la zona en que vive.

Sería muy recomendable encontrar la forma de obtener semillas bien desarrolladas a fin de poder propagar estas plantas a nivel comercial, tanto en su región nativa, como en otras localidades con condiciones ambientales similares.

El crecimiento de los árboles es relativamente rápido, aunque al parecer no tan rápido como el de Liquidambar, con el que tan frecuentemente convive. 
Afinidades de la especie

La mayor parte de los ejemplares de $D$. palo-escrito, previamente depositados en los herbarios, habían sido identificados como $D$. tucurensis Donn. Smith y es probable que de hecho se trate de una especie emparentada. Ambas comparten los caracteres de estandarte erecto y estilo grueso, androceo de 9 estambres, flores de 3 a $5.5 \mathrm{~mm}$ de largo, inflorescencia paniculada, foliolos relativamente grandes en número próximo a 11, fruto monospermo, similar en la forma, y en ser (al menos potencialmente) árboles altos de ambientes húmedos.

No obstante, $D$. tucurensis tiene los foliolos con muy marcada tendencia a ser oblongos y provistos de pubescencia amarilla conspicua en el envés, mientras en $D$. palo-escrito éstos son casi siempre francamente ovados y si bien, son bicolores de manera evidente, carecen por completo de tonalidad amarilla en la pubescencia. El fruto de esta última es pubescente, en cambio la primera lo tiene glabro. En $D$. tucurensis. además, el cáliz presenta venación muy prominente por dentro, carácter que no se observa en $D$. palo-escrito. $D$. tucurensis se describió de Guatemala y se conoce en la actualidad de Chiapas y Belice a Nicaragua.

Otras especies afines son $D$. melanocardium Pittier, $D$. congestiflora Pittier y $D$. glomerata Hemsl. La primera y la segunda tienen los foliolos mucho más pequeños, en la tercera el fruto es glabro y con frecuencia lleva 2 ó 3 semillas.

\section{AGRADECIMIENTOS}

El Ing. Mauro Márquez G. brindó valiosa ayuda en la obtención del material necesario para la descripción anatómica de la madera. La Dra. Velva E. Rudd tuvo la amabilidad de leer criticamente el borrador del manuscrito. El Dr. Mario Sousa proporcionó material bibliográficó. EI Sr. Rodrigo Tavera es el autor de la ilustración de la planta. El Biól. Sergio Zamudio realizó colectas específicas de material de herbario. Se agradece cumplidamente todo este apoyo recibido.

\section{LITERATURA CITADA}

Bentham, G. 1860. Synopsis of the Dalbergieae, a tribe of Leguminosae. Journ. Proc. Linn. Soc., Bot. 4 (Suppl.): $1-134$.

Dwyer, J.D. 1965. Dalbergia, in Flora of Panama, part V, fascicle 4. Ann. Mo. Bot. Gard. 52: 398-403.

Guridi Gómez, L.I. 1980. La madera en las artesanías del estado de Michoacán. Instituto Nacional de Investigaciones Forestales. Bol. Divulg. 50. México, D.F. $131 \mathrm{pp}$.

Pittier, H. 1922. On the species of Dalbergia of Mexico and Central America. Journ. Wash. Acad. Sci. 12: 54-64.

Record, S.J. \& R.W. Hess. 1943. Timbers of the New World. Yale University Press. New Haven. 640 pp.

Rzedowski, J. 1965. Vegetación del estado de San Luis Potosí. Acta Cient. Potos. 5: 5-291.

Rzedowski, J. 1966. Nombres regionales de algunas plantas de la Huasteca Potosina. Acta Cient. Potos. 6: 7-58.

Standley, P.C. 1922. Amerimnon, in Trees and shrubs of Mexico. Contr. U.S. Nat. Herb. 23: 506-508.

Standley, P.C. 1937. Dalbergia, in Flora of Costa Rica. Field Mus. Nat. Hist. Bot. Ser. 18: 531-532.

Standley, P.C. \& J.A. Steyermark. 1946. Dalbergia, in Flora of Guatemala. Fieldiana: Botany 24(5): 201208. 